\title{
COVID-19 in a Patient with Liver Cirrhosis
}

\author{
Edita Kreivenaite $^{1}$, Rolandas Gedgaudas ${ }^{1}$, Irena Valantiene ${ }^{1}$, Aukse Mickiene ${ }^{2}$, Juozas Kupcinskas ${ }^{1}$
}

1) Lithuanian University of Health Sciences, Medical Academy, Department of Gastroenterology, Kaunas 2) Lithuanian University of Health Sciences, Medical Academy, Department of Infectious Diseases, Kaunas, Lithuania
Address for correspondence: Juozas Kupcinskas Lithuanian University of Health Sciences, Medical Academy,

Department of Gastroenterology \& Institute for Digestive Research, 9a Mickeviciaus St., Kaunas, LT-44307, Lithuania juozas.kupcinskas@lsmuni.lt
Received: 24.04.2020

Accepted: 14.05.2020

\begin{abstract}
The outbreak of coronavirus disease 2019 (COVID-19) has recently become a serious issue affecting thousands of people worldwide. It is known that a substantial proportion of patients infected with COVID-19 have abnormal liver function tests; however, the consequences of this information is still not clear. Here we present the first case report of a patient with liver cirrhosis and COVID-19 in our centre. Resolution of COVID-19 symptoms was observed after six days of fever onset. We observed only slight fluctuations of liver enzymes, bilirubin levels and INR without clinical consequences in our case. We suggest testing for severe acute respiratory syndrome coronavirus on any cirrhotic patient on initial presentation, even without symptoms of COVID-19 in areas where the epidemic was prevalent.
\end{abstract}

Key words: liver cirrhosis - chronic viral hepatitis - coronavirus disease 2019.

Abbreviations: ACE: angiotensin-converting enzyme; ALT: alanine aminotrasferase; AST: aspartate aminotransferase; COVID-19: coronavirus disease 2019; CRP: C-reactive protein; CT: computed tomography; GGT: gamma glutamyl transferase; INR: international normalised ratio; MELD: Model for End-Stage Liver Disease; SARS-CoV-2: severe acute respiratory syndrome coronavirus 2.

\section{INTRODUCTION}

Coronavirus disease 2019 (COVID-19) has become a huge challenge for global public health with the number of confirmed cases and deaths growing daily. The pandemic has raised the concern that patients with liver cirrhosis might be more susceptible to severe acute respiratory syndrome coronavirus 2 (SARSCoV-2) infection due to their systemic immunocompromised status $[1,2]$. On the contrary, according to the available data, immunosuppression may even provide some protection against hyperactivated immune response $[3,4]$. Published data suggest that a substantial proportion of patients infected with COVID-19 have abnormal liver function tests $[1,5]$. However, mechanisms of liver injury induced by COVID-19 and clinical consequences of these alterations remain unclear [6, 7]. Recently, a COVID-19 and liver disease registry was launched [8]; nevertheless, there is still scarcity of data on how COVID-19 affects patients with pre-existing liver disease.

\section{CASE REPORT}

On March 16, 2020, a 48-year-old female with a history of hypertension, obesity and cirrhosis induced by active hepatitis C virus infection (Child-Pugh 8 points, MELD-Na 13 points), was admitted to the hospital due to melena. On her physical examination vitals were stable with no clinical signs of overt hepatic encephalopathy or fever. On admission, abdominal ultrasound showed liver cirrhosis with mild ascites. Laboratory tests demonstrated elevated C-reactive protein (CRP) (23 $\mathrm{mg} / \mathrm{l})$, slightly elevated total bilirubin $(41.2 \mu \mathrm{mol} / \mathrm{l})$, aspartate aminotransferase (AST) (49 IU/l), gamma glutamyl transferase (GGT) (62 IU/l) levels and international normalised ratio (INR) 1.5. Diagnosis of spontaneous bacterial peritonitis was excluded after ascites puncture. Endoscopy demonstrated grade II oesophageal varices and duodenal ulcer with no signs of active bleeding (Forrest class III). A second endoscopy with oesophageal variceal ligation was performed. Medical therapy of the patient consisted of ciprofloxacin prophylaxis, proton pump inhibitors and non-selective beta-blockers. The patient 


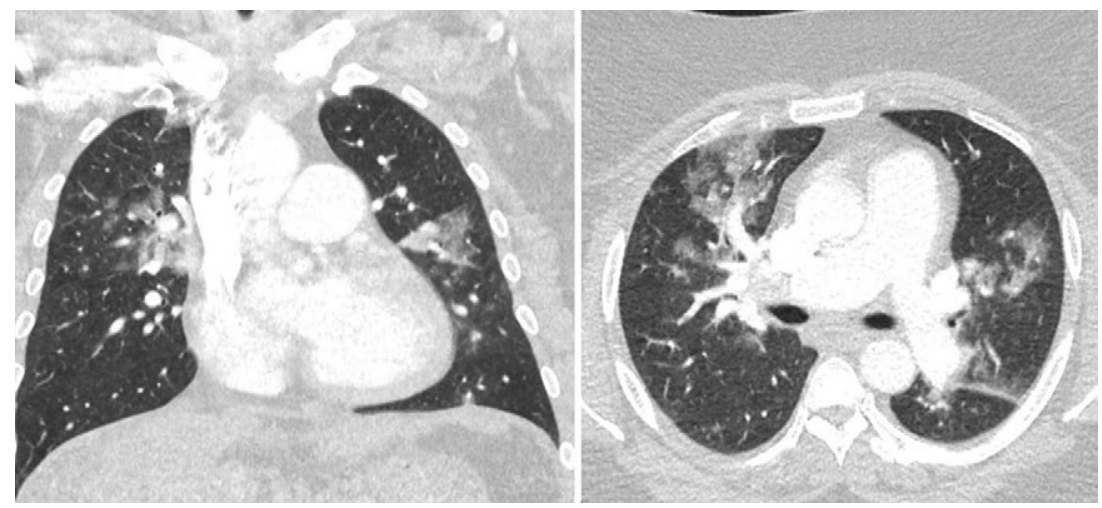

Fig. 1. A chest CT scan showing ground-glass opacity pattern bilaterally more intense in the upper lobes.

was discharged after four days of hospitalization but returned to emergency department on the same evening with a fever of $39^{\circ} \mathrm{C}$. Interestingly, no initial signs of infection were observed on the chest $\mathrm{x}$-ray, ascites or urine analysis. On readmission CRP was $42.8 \mathrm{mg} / \mathrm{l}$, blood culture was drawn, and empirical antibiotic therapy was initiated. Other laboratory tests did not show any clinically significant changes except moderate increase in AST level (57 IU/1). Over the next two days fever up to $39^{\circ} \mathrm{C}$ remained and was accompanied by progressive dyspnoea requiring supportive oxygen therapy; therefore, a nasopharyngeal swab was taken and the presence of SARSCoV-2 was confirmed. A further computed tomography (CT) scan of chest revealed bilateral ground-glass opacity pattern (Fig. 1). The patient was transferred to a specialized infectious disease unit for further care. By the 6th day of COVID-19 disease development, symptoms had resolved and there was no need for additional oxygen. After two consecutive negative COVID-19 tests the patient was discharged. On the day of discharge laboratory tests showed elevated total bilirubin (46.1 $\mu \mathrm{mol} / \mathrm{l})$, AST (49 IU/l), GGT (48 IU/l) and INR (1.6).

\section{DISCUSSION}

We hereby report the first case of a COVID-19 pneumonia in a liver cirrhosis patient in our centre. When evaluating the dynamics of liver enzymes and function tests before and after the patient was diagnosed with COVID-19, we observed only slight fluctuations of total bilirubin, AST and INR with no clinical consequences in our case.

Varying degrees of liver injury have been observed in patients with COVID-19. Recent studies have shown that patients with COVID-19 had elevation of AST or ALT in people faring the worst [9], with slightly elevated bilirubin levels in some cases $[9,10]$. Although in most cases elevation of liver enzymes was mild-to-moderate, one case of acute hepatitis (AST 1230 IU/l; ALT 697 IU/l) before the development of respiratory symptoms was recently published [11]. Moreover, Weber et al. [12] recently presented a case of severe liver failure illustrated by MELD 40 without pre-existing liver disease. In addition to this, a case report of an acute liver failure (AST 741 U/l; ALT 241 IU/l, INR 2.6) in a patient with decompensated alcoholic cirrhosis was described [13]. However, currently there is not enough data on other complications in patients with cirrhosis and COVID -19, therefore further research is needed.
Although the mechanism of liver injury from SARS-CoV-2 remains unclear, the possible reason for elevation of liver enzymes could be a direct effect of the virus to the liver using angiotensin-converting enzyme 2 (ACE2) receptors [14]. Previous studies have shown that ACE2 receptors are expressed in both bile duct cells and liver cells, but concentration of ACE2 receptors in hepatocytes is much lower and for this reason liver injury may be due to the damage of cholangiocytes [15]. However, since cholestatic liver enzymes are not usually elevated in COVID-19 patients with liver damage and histopathologic liver features of patients with COVID-19 did not show any remarkable damage in neither hepatocytes nor cholangiocytes [16], liver injury could be induced by secondary causes. An additional cause of liver impairment in patients with COVID-19 may be a hyperactivated immune response and systemic inflammation due to cytokine storm or drug hepatotoxicity [17]. Therefore, more intensive surveillance of the patients and close monitoring of liver function is warranted.

Experts suggest that the elevation of liver enzymes might present clinical challenges especially to patients with pre-existing liver disease [17]. Patients with liver cirrhosis are expected to be at risk of a severe infectious disease course [4]. Data suggest that in patients with liver cirrhosis more severe courses of influenza were seen compared with patients without liver disease [18]. Moreover, in a study in Gastroenterology by Singh and Khan [19] where outcomes of the patients with pre-existing liver disease and without liver disease were compared, investigators found that patients with underlying liver disease, notably cirrhosis, had a significantly higher risk of mortality. In our case the patient's fever and dyspnoea improved within several days without any specific treatment and a follow-up chest $\mathrm{x}$-ray on the 11th day after diagnosis of COVID-19 showed no signs of lung infiltration. Moreover, considering a COVID-19 incubation time of up to 14 days, it is possible that the patient was already harbouring SARS-CoV-2 when he was admitted for the first time with no clinical signs of infection.

\section{CONCLUSION}

Based on this case we suggest to test any cirrhotic patient for SARS-CoV-2 on initial presentation, even without symptoms of COVID-19 in areas where an epidemic is prevalent. Overall, further data are required to investigate the outcomes and 
best treatment strategies in patients with liver cirrhosis and COVID-19.

Conflicts of interest: None to declare.

Authors' contribution: E.K., J.K. conceived and wrote the manuscript. R.G. managed the patient. R.G., I.V., A.M analysed and reviewed the data. J.K. drafted the work. All authors approved the final version of the manuscript.

\section{REFERENCES}

1. Jin X, Lian JS, Hu JH, et al. Epidemiological, clinical and virological characteristics of 74 cases of coronavirus-infected disease 2019 (COVID-19) with gastrointestinal symptoms. Gut 2020;69:1002-1009. doi:10.1136/gutjnl-2020-320926

2. Zhang C, Shi L, Wang FS. Liver injury in COVID-19: management and challenges. Lancet Gastroenterol Hepatol 2020;5:428-430. doi:10.1016/ s2468-1253(20)30057-1

3. D'Antiga L. Coronaviruses and immunosuppressed patients: The facts during the third epidemic. Liver Transpl 2020 Mar 20. doi:10.1002/lt.25756

4. Boettler T, Newsome PN, Mondelli MU, et al. Care of patients with liver disease during the COVID-19 pandemic: EASL-ESCMID Position Paper. JHEP Rep 2020;2:100113. doi:10.1016/j.jhepr.2020.100113

5. Chen N, Zhou M, Dong X, el al. Epidemiological and clinical characteristics of 99 cases of 2019 novel coronavirus pneumonia in Wuhan, China: a descriptive study. Lancet 2020;395:507-513. doi:10.1016/S0140-6736(20)30211-7

6. Li J, Fan JG. Characteristics and mechanism of liver injury in 2019 coronavirus disease. J Clin Transl Hepatol 2020;8:13-17. doi:10.14218/ JCTH.2020.00019

7. Ong J, Young BE, Ong S. COVID-19 in gastroenterology: a clinical perspective. Gut 2020;69:1144-1145. doi:10.1136/gutjnl-2020-321051

8. EASL. COVID 19 in patients with liver disease or transplantation. COVIDHep registry. Available from: http://covid-hep.net. Accessed 10 April 2020.
9. Guan WJ, Ni ZY, Hu Y, et al. Clinical characteristics of coronavirus disease 2019 in China. N Engl J Med 2020;382:1708-1720. doi:10.1056/ NEJMoa2002032

10. Xu L, Liu J, Lu M, Yang D, Zheng X. Liver injury during highly pathogenic human coronavirus infections. Liver Int 2020;40:998-1004. doi:10.1111/liv.14435

11. Wander P, Epstein M, Bernstein D. COVID-19 Presenting as Acute Hepatitis. Am J Gastroenterol 2020 Apr 15. doi:10.14309/ ajg.0000000000000660

12. Weber S, Mayerle J, Irlbeck M, Gerbes AL. Severe liver failure during SARS-CoV-2 infection. Gut 2020 Apr 23. doi:10.1136/ gutjnl-2020-321350

13. Qiu H, Wander P, Bernstein D, Satapathy SK. Acute on Chronic Liver Failure from Novel Severe Acute Respiratory Syndrome Coronavirus 2 (SARS-CoV-2). Liver Int 2020 May 5. doi:10.1111/liv.14506

14. Hoffmann M, Kleine-Weber H, Krüger N, Müller M, Drosten C, Pöhlmann S. The novel coronavirus 2019 (2019-nCoV) uses the SARS-coronavirus receptor ACE2 and the cellular protease TMPRSS2 for entry into target cells. Biorxiv 2020.01.31.929042. doi:10.1101/2020.01.31.929042

15. Chai X, Hu L, Zhang Y, et al. Specific ACE2 expression in cholangiocytes may cause liver damage after 2019-nCoV infection. BioRxiv 2020.02.03.931766. doi:10.1101/2020.02.03.931766

16. Xu Z, Shi L,Wang Y, et al. Pathological findings of COVID-19 associated with acute respiratory distress syndrome. Lancet Respir Med 2020;8:420-422. doi:10.1016/s2213-2600(20)30076-x

17. Feng G, Zheng KI, Yan QQ, et al. COVID-19 and liver dysfunction: Current insights and emergent therapeutic strategies. J Clin Transl Hepatol 2020;8:18-24. doi:10.14218/JCTH.2020.00018

18. Schütte A, Ciesek S, Wedemeyer H, Lange CM. Influenza virus infection as precipitating event of acute-on-chronic liver failure. J Hepatol 2019;70:797-799. doi:10.1016/j.jhep.2018.11.015

19. Singh S, Khan A. Clinical Characteristics and Outcomes of COVID-19 Among Patients with Pre-Existing Liver Disease in United States: A Multi-Center Research Network Study. Gastroenterology 2020 May 3. doi:10.1053/j.gastro.2020.04.064 